\title{
Glucose Metabolism, Lactate, and Ammonia Production by the Human Placenta In Vitro
}

\author{
IAN R. HOLZMAN, ANTHONY F. PHILIPPS, AND FREDERICK C. BATTAGLIA ${ }^{(27)}$ \\ Division of Perinatal Medicine, Departments of Pediatrics, and Obstetrics and Gynecology, University of Colorado \\ Medical Center, Denver, Colorado, USA
}

\section{Summary}

Fifteen human placentas were obtained at term. Placental fragments were incubated in a bicarbonate-buffered Earle's solution. Additions of glutamate $(1 \mathrm{mM})$ or glutamine $(1$ or $2 \mathrm{mM})$ were made. All incubations showed a net utilization of glucose. There was a striking variability among placentas in the net glucose utilization rate $(1.27 \mu$ moles $/ \mathrm{g} / \mathrm{hr}-11.44 \mu \mathrm{moles} / \mathrm{g} / \mathrm{hr}$, coefficient of variation $=62 \%$ ). The intraplacental coefficient of variation in glucose utilization was only $14 \%$. All placental incubations showed a net production of both lactate (mean $=7.5 \mu$ moles $/ g$ placental wet weight $/ \mathrm{hr}$ ) and ammonia (mean $=3.5 \mu$ moles $/ g$ placental wet weight/hr). There was no correlation between lactate or ammonia production and glucose utilization. The addition of sodium glutamate $(1 \mu \mathrm{mole} / \mathrm{ml})$ produced no change in glucose utilization or ammonia production. The addition of glutamine (1 and $2 \mu \mathrm{moles} /$ $\mathrm{ml}$ ) produced a significant increase in ammonia production over that found in the controls, but no change in glucose utilization. Incubation with $2 \mu$ moles glutamine/ $\mathrm{ml}$ demonstrated an increase in lactate production. All incubations showed a striking increase in ammonia concentration after $\mathbf{4 5}$ min of incubation.

Individual placentas may differ markedly in their ability to utilize glucose in an in vitro system. Ammonia production may be a normal metabolic endproduct in a tissue lacking an active urea cycle or a byproduct of the purine nucleotide cycle.

\section{Speculation}

The marked variability in glucose utilization by the in vitro human placenta may be an important descriptive characteristic of the normal term placenta that is related to functional differences among apparently similar placentas.

The human placenta produces ammonia in vitro at an appreciable rate. A high rate of ammonia and lactate production under aerobic conditions may be a general property of trophoblast.

Studies of human placental metabolism have included investigations of the placenta's ability to use and store carbohydrates ( 5 , $17,20,24)$ and to transport amino acids $(4,22)$.

These studies have shown considerable variability in some metabolic characteristics, such as glucose utilization rate. In addition, recent in vivo steady-state studies of the ovine placenta have suggested an important role for placental lactate and ammonia production in fetal metabolism $(3,9)$. The present study was undertaken to determine whether interplacental variability in glucose utilization would persist among placentas carefully selected to be of comparable gestational age and free of pathology. In addition, the ability of the human placenta to produce lactate and ammonia under aerobic conditions was studied for purposes of interspecies comparison.

\section{MATERIALS AND METHODS}

\section{TISSUE PREPARATION}

Fifteen placentas were obtained at the time of vaginal delivery from women with term, uncomplicated pregnancies. Five of the placentas were used solely for amino acid analysis. However, all placentas were handled in the same manner for tissue preparation. The placentas were placed on ice immediately, and a wedge of tissue was taken from an area approximately one-half the distance between the placental margin and the insertion of the umbilical cord. Fifteen to 20 fragments, each weighing $250-650 \mathrm{mg}$, were removed from this wedge, with care being taken to select tissue at approximately one-third the distance from the membranes to the maternal surface. Each fragment was teased loose and washed in normal saline solution at $4^{\circ}$ until free of all visible blood. The fragments were then placed in fresh, iced saline to await incubation, which was begun immediately after preparation of the samples. The total time from delivery to incubation was 30-45 min.

\section{TISSUE INCUBATION}

A bicarbonate-buffered Earle's solution (Microbiological Associates, Inc., Los Angeles, CA) was diluted to contain $100 \mathrm{mg}$ glucose/dl. Four $\mathrm{ml}$ of this solution, were placed in $25-\mathrm{ml}$ flasks and equilibrated with a gas mixture containing $5 \% \mathrm{CO}_{2}, 95 \% \mathrm{O}_{2}$. Test flasks contained either sodium glutamate $(1 \mathrm{mM})$ or glutamine ( 1 or $2 \mathrm{mM}$ ). A wet weight of the tissue fragments was obtained before incubation. Fragments with wet weights of $300-500 \mathrm{mg}$ were placed in the test and control flasks, which were then reequilibrated with the gas mixture and stoppered tightly. The flasks were incubated in a metabolic shaker at $37^{\circ}$ for $45 \mathrm{~min}$. Four or five separate control and test incubations were performed for each placenta.

At the beginning of the incubation period, other placental fragments with a total weight of approximately $1 \mathrm{~g}$ were weighed, added to $6 \mathrm{ml}$ cold, demineralized water, and homogenized with a glass tissue homogenizer for at least $1 \mathrm{~min}$. The homogenates were centrifuged for $10 \mathrm{~min}$ at $4^{\circ}$, placed on ice, and analyzed for ammonia immediately. Analyses for amino acids, lactate, and glucose were made within $2 \mathrm{hr}$. Initially, four to five fragments from different areas of each placenta were analyzed separately to check for intraplacental variations in amino acid content. Since no significant differences were found, the data were pooled for each placenta.

After $\mathbf{4 5}$ min of incubation, the tissue fragments were removed, homogenized, centrifuged, and analyzed in the same manner as the unincubated fragments. In six experiments designed to determine the net change in free amino acids, the tissues were homogenized directly in their incubation solutions. The medium was centrifuged for $10 \mathrm{~min}$ at $4^{\circ}$ and used directly for chemical determinations. 


\section{CHEMICAL METHODOLOGY}

Glucose concentrations in both tissue and incubation media were determined by the glucose oxidase method (Beckman Instruments, Inc.). Glucose utilization was calculated as the difference between glucose concentrations in tissue and medium before and after the $45 \mathrm{~min}$ incubation. Glutamine and glutamate were measured according to the fluoroenzymatic method of Nahorski (16). In those experiments in which the total free amino acid pool was determined, the values for glutamine and glutamate are those measured by column chromatography. A comparison of the two methods demonstrated good agreement among results from samples obtained under similar conditions. Ammonia was analyzed according to the ion-exchange method of Kurahasi et al. (11). Lactate was determined according to the enzymatic method of Olsen (18). Ammonia and lactate production rates were calculated as the difference between the levels present in tissue and medium before and after incubation.

Amino acids were analyzed using a two-column JEOL-6AH amino acid analyzer and buffer systems as described previously (12). Fresh solution was prepared every $10-14$ days, and $100-\mu \mathrm{M}$ standards of the amino acids (Pierce Chemical Co., Rockford, IL) were analyzed in triplicate at the beginning and end of each batch of ninhydrin solution. The coefficients of variation in optical densities within sets of three standards analyzed in a 36-hr run were $2 \%$ or less for all amino acids. In order to avoid the interference of glutathione with the analysis of aspartic acid, glycine, and alanine, one-half of the sample collection from each homogenate was treated with performic acid as previously described (13).

\section{RESULTS}

All incubations in Earle's solution containing $100 \mathrm{mg}$ glucose/ dl showed a net utilization of glucose (Fig. 1). However, there was a striking variability among placentas in the net glucose utilization rate, with a range from $1.27 \mu$ moles $/ \mathrm{g} / \mathrm{hr}$ to $11.44 \mu \mathrm{moles} / \mathrm{g} / \mathrm{hr}$ and a coefficient of variation of $62 \%$. In sharp contrast to the interplacental variability, the mean variance in the glucose utilization rate within tissue samples from the same placentas was only $14 \%$. The striking variability in glucose utilization between placentas could not be correlated with differences in the length of time elapsing from birth until incubation nor with gross morphologic differences between placentas. All placental incubations showed a net production of both lactate (mean $=7.5 \mu$ moles $/ \mathrm{g}$ placental wet weight $/ \mathrm{hr}$ ) and ammonia (mean $=3.5 \mu$ moles $/ \mathrm{g}$ placental wet weight/hr) (Fig. 1). A calculation of the correlation between lactate production and glucose utilization for each incubation $(r=+0.3077, P>0.1)$ and ammonia production and glucose utilization $(r=-0.1291, P>0.1)$, revealed no significant correlation between these events.

The addition of sodium glutamate to the incubation medium at a final concentration of $1 \mu \mathrm{mole} / \mathrm{ml}$ produced no change in glucose utilization or ammonia production. A small, but statistically significant, increase in lactate production was noted (Table 1). The addition of glutamine at a final concentration of both 1 and $2 \mu \mathrm{moles} / \mathrm{ml}$ produced a significant increase in ammonia production over that found in the controls. In neither set of incubations was there any change in glucose utilization. The placental fragments incubated in the solution containing $2 \mu$ moles glutamine/ml did show a small but significant increase in lactate production (Table 1). Data from the incubation in which the solution contained $2 \mu$ moles glutamine $/ \mathrm{ml}$ are shown in Figure 2. There was a stoichiometric relationship between the increase in ammonia and glutamate production and the utilization of glutamine. All incubations showed a striking increase in ammonia concentration after $45 \mathrm{~min}$ of incubation.

Analysis of the pool of free amino acids demonstrated a significant increase in concentration of leucine, valine, threonine, phenylalanine, tyrosine, and histidine over the 45-min incubation (Fig. 3). Glutamine was the only amino acid which appeared to be used during incubation, but the decrease in glutamine levels was not statistically significant.

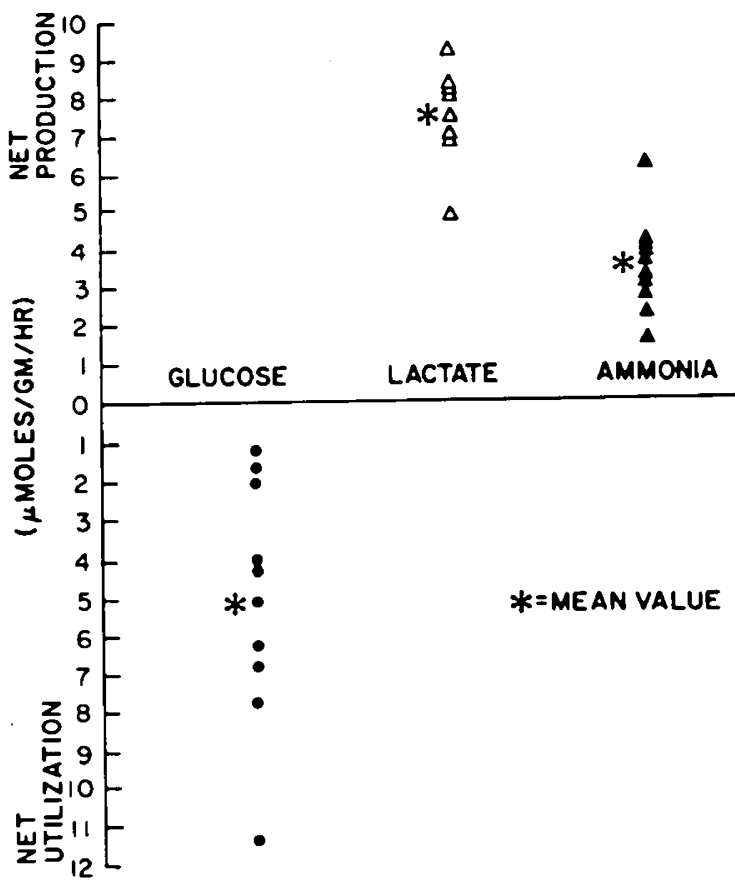

Fig. 1. The utilization of glucose and the production of lactate and ammonia by human placental tissue fragments from separate placentas at term. Each data point represents the average of four to five separate incubations on each individual placenta.

Table 1. Effects of glutamine and glutamate on the utilization of glucose and the production of lactate and ammonia

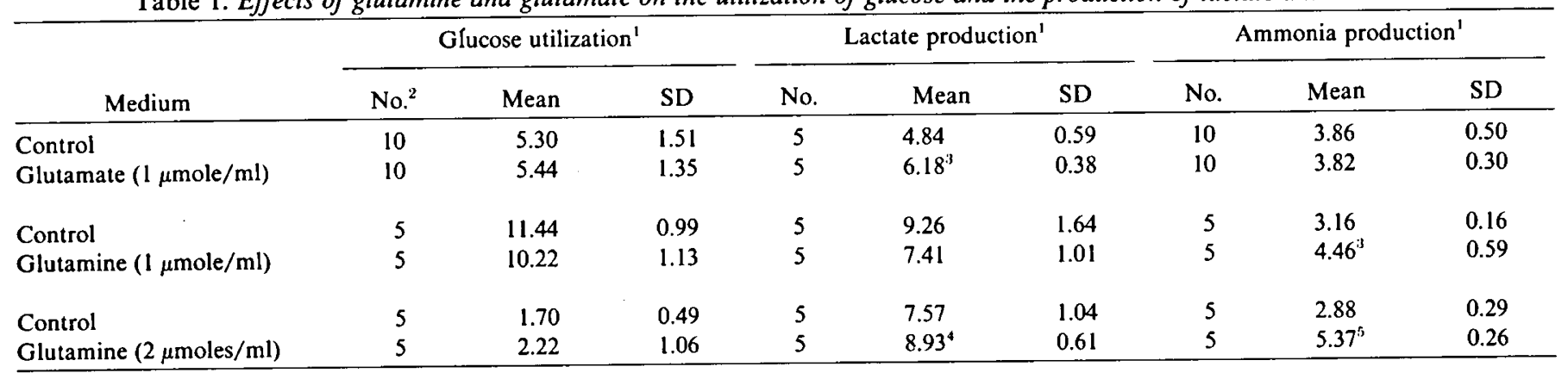

\footnotetext{
' $\mu$ moles/g/hr.

${ }^{2}$ Number of flasks incubated, in each study all tissue fragments for control and experimental groups obtained from the same placenta.

${ }^{3} P<0.01$.

${ }^{4} P<0.05$.

${ }^{5} P<0.001$.
} 


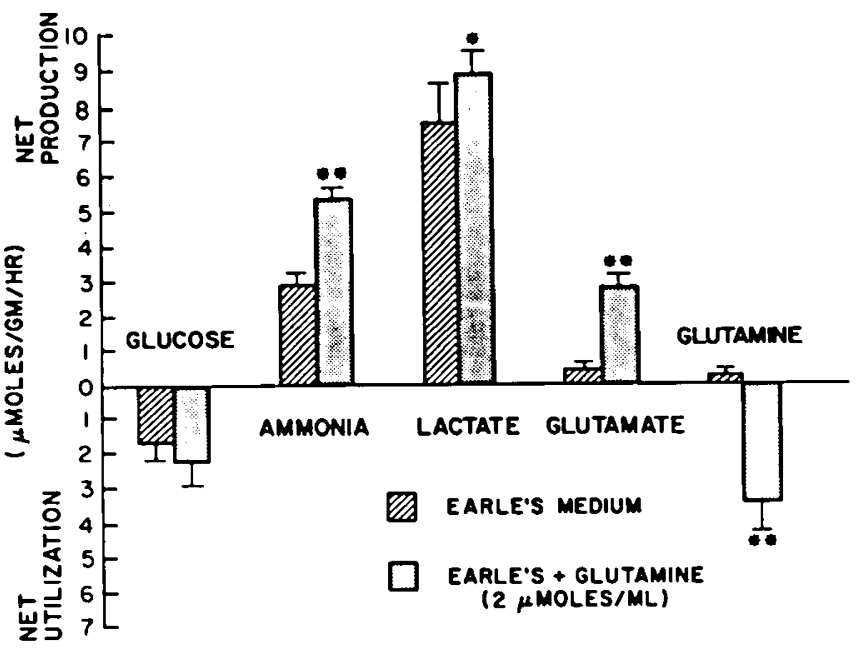

Fig. 2. A representative incubation of human placental tissue fragments obtained from the same placenta demonstrating the effects of the addition of glutamine to the incubation solution at a final concentration of 2 $\mu \mathrm{moles} / \mathrm{ml}$. Statistical significance between control and test incubations by two-tailed $t$-test, ${ }^{*} P<0.05,{ }^{*} P<0.001$. Each bar represents the mean \pm SD of five separate incubations.

\section{DISCUSSION}

Villee (24) reported glucose utilization rates by term human placentas in vitro, in which large interplacental differences in glucose utilization were noted. In vitro placental perfusion studies have also noted a 4-fold difference in glucose utilization rate between placentas $(17,20)$. The data presented in this study are similar to those of Villee (24) and support the concept that individual placentas may differ markedly in their ability to utilize glucose in an in vitro system.

The intraplacental variability in glucose consumption was only $14 \%$ as compared with the variability between placentas of $62 \%$. This suggests that the differences reflect biologic variability in metabolism among placentas rather than simply differences in the tissue samples obtained.

Although there was no correlation between glucose utilization and lactate production, the molar ratio of lactate produced to glucose utilized (1.5:1.0) fell within the range of values reported for tissue slice (24) and perfusion (17) preparations. The suggestion that lactate production is not directly linked to glucose utilization is consistent with the work of Garber et al. (8) on the rat epitrochlearis muscle preparation. Those workers noted that only $20-40 \%$ of the lactate and pyruvate released could be accounted for by glucose utilization. They suggested that amino acid metabolism was a likely source for production of the remainder of the lactate and pyruvate.

The production of free ammonia by nonrenal tissues has been demonstrated in a number of in vitro studies $(2,6,21,25)$. The placenta of the pregnant sheep, in vivo, has also been shown to produce ammonia in significant quantities (9). In 1951, Luschinsky (15) demonstrated the ability of human placental homogenates and extracts to desamidate glutamine and thus produce ammonia. Further work by Adachi (1) has suggested the possibility of a placental glutaminase with concomitant ammonia production. The addition of glutamine to the incubation medium in the present study produced a stoichiometric increase in the amount of ammonia present in the system. Although glutamine is known to decompose spontaneously, the incubation of glutamine in Earle's solution without placental tissue did not yield an increase of ammonia during the $\mathbf{4 5} \mathrm{min}$ incubation.

When glutamate was added to the incubation medium, no change in ammonia production occurred. This may simply reflect one of the major problems associated with in vitro placental research. It is likely that the major placental surface exposed to

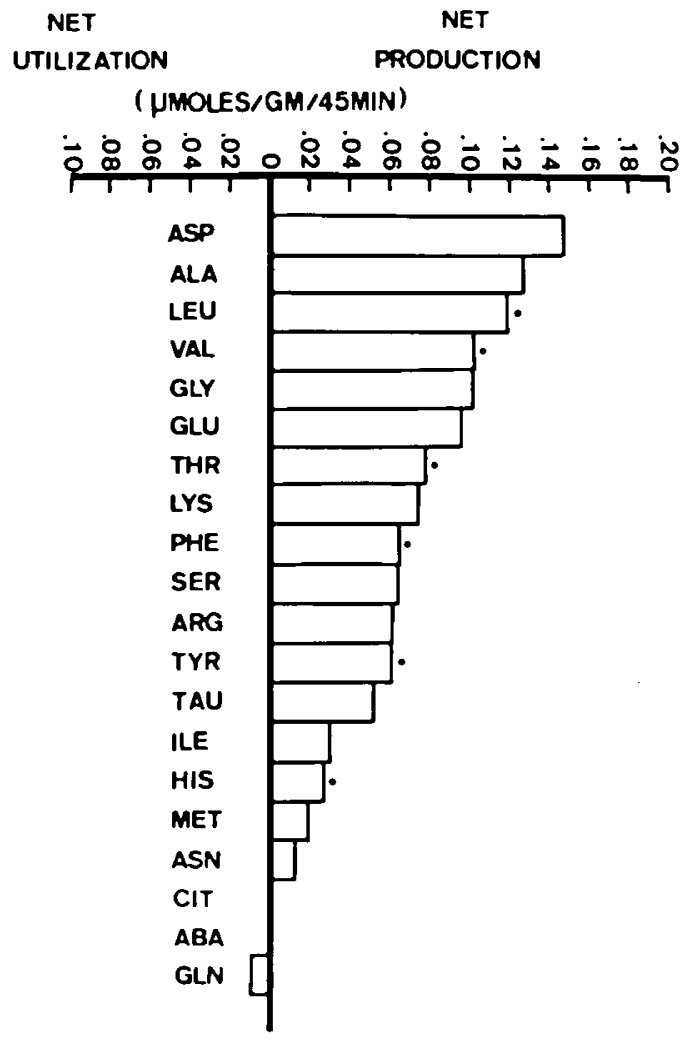

Fig. 3. The changes in the pool of free amino acids in human placental tissue fragments occurring during the 45 -min incubation. Those changes which were significantly $(P<0.05)$ different from zero are denoted by an asterisk.

the medium is the maternal surface. Experiments in the sheep (9), rat (7), and monkey (23) have suggested that glutamate is not easily transferred from the maternal circulation into the placenta. Even if some glutamate is transported into the placenta tissue, the large extracellular water space $(>50 \%)$ can lead to inaccuracies in calculating the amount of substrate that is actually intracellular. When the appropriate calculation for estimated water distribution was performed in the present study, the major portion of glutamate measured in the tissue was present in the extracellular fluid. Thus, if the maternal surface of the human placenta does not readily transport glutamate intracellularly, it is not surprising that the addition of glutamate to the medium had no apparent effect upon ammonia production.

The general increase in the free amino acid pool size within the placental fragments is consistent with protein hydrolysis of sufficient magnitude to account for the ammonia production.

Although it is possible that the ammonia production observed in this study is purely a reflection of proteolysis, this seems unlikely. Ammonia production has been shown to be alterable in a number of in vitro preparations: changes in carbohydrate substrate affect ammonia production by the mouse sarcoma (21), rat spleen (6), and Jensen rat sarcoma (6). In vivo changes in keto acid concentration affect placental ammonia production (1). Placental ammonia production has been shown for the ovine placenta (9). It is more likely that ammonia may be a normal metabolic endproduct in a tissue lacking an active urea cycle. Although the human placenta appears to possess glutaminase-like activity, the role of this pathway in the production of ammonia is unknown. It is equally possible that the ammonia production observed in this study could be accounted for by the presence of a purine nucleotide cycle in which aspartate and inosine monophosphate are converted to fumarate and adenosine monophosphate (14). Data concerning the functioning of this cycle, or the presence of the requisite enzymes in the human placenta, are not yet available. 


\section{CONCLUSION}

In vitro incubations of term human placentas demonstrated a large interplacental variability in glucose utilization rates. Lactate and ammonia production could be demonstrated under aerobic conditions for all placentas studied. Ammonia production could be increased by the addition of glutamine to the incubation medium, but not by the addition of glutamate. Neither lactate nor ammonia production were related to the glucose utilization rate.

\section{REFERENCES AND NOTES}

1. Adachi, H.: The placenta and hormones. J. Jpn. Obstet. Gynecol. Soc., 19: 665 (1967).

2. Benjamin, A. M., and Quastel, J. H.: Metabolism of amino acids and ammonia in rat brain cortex slices in vitro: A possible role of ammonia in brain function. J. Neurochem., 25: 197 (1975).

3. Burd, L. I., Jones, M. D., Simmons, M. A., Makowski, E. L., Meschia, G., and Battaglia, F. C.: Placental production and foetal utilization of lactate and pyruvate. Nature, 254: 710 (1975).

4. Dancis, J., Money, W. L., Springer, D., and Levitz, M.: Transport of amino acids by placenta. Am. J. Obstet. Gynecol., 101: 820 (1968).

5. Diamant, Y. Z., Mayorek, N., Neuman, S., and Shafrir, E.: Enzymes of glucose and fatty acid metabolism in early and term human placenta. Am. J. Obstet. Gynecol., 121: 58 (1975).

6. Dickens, F., and Greville, G. D.: CL. Metabolism of normal and tumour tissue. IX. Ammonia and urea formation. Biochem. J., 27: 1123 (1933).

7. Dierks-Ventling, C., Cone, A. L., and Wapnir, R. A.: Placental transfer of amino acids in the rat. I. L-glutamic acid and L-glutamine. Biol. Neonate, 17: 361 (1971).

8. Garber, A. J., Karl, I. E., and Kipnis, D. M.: Alanine and glutamine synthesis and release from skeletal muscle. I. Glycolysis and amino acid release. J. Biol. Chem., 251: 826 (1976).

9. Holzman, I. R., Lemons, J. A., Meschia, G., and Battaglia, F. C.: Ammonia production by the pregnant uterus. Proc. Soc. Exp. Biol. Med., 156: 27 (1077)

10. Kodama, I.: Studies on ammonia metabolism of placenta. J. Jpn. Obstet. Gynecol. Soc., 24: 155 (1972).

11. Kurahasi, K., Ishihara, R., and Uehara, H.: Enzymatic determination of ammonia in blood plasma. Clin. Chim. Acta, $42: 141$ (1972).
12. Lemons, J. A., Adcock, E. W. III, Jones, M. D. Jr., Naughton, M. A., Meschia, G., and Battaglia, F. C.: Umbilical uptake of amino acids in the unstressed fetal lamb. J. Clin. Invest., 58: 1428 (1976).

13. Lemons, J. A., Teng, C., and Naughton, M. A.: Removal of glutathione interference in blood amino acid analysis. Biochem. Med., 15: 282 (1976)

14. Lowenstein, J. M.: Ammonia production in muscle and other tissues: the purine nucleotide cycle. Physiol. Rev., 52: 382 (1972).

15. Luschinsky, H. L.: The activity of glutaminase in the human placenta. Arch. Biochem. Biophys., 31: 132 (1951).

16. Nahorski, S. R.: Fluorometric measurement of glutamine and asparagine using enzymatic methods. Anal. Biochem., 42: 136 (1971).

17. Nesbitt, R. E. L. Jr., Rice, P. A., and Rourke, J. E.: In vitro perfusion studies of the human placenta. III. The relationships between glucose utilization rates and concentration. Gynecol. Invest., 4: 243 (1973).

18. Olsen, C.: An enzymatic fluorometric micromethod for the determination of acetoacetate, $\beta$-hydroxybutyrate, pyruvate and lactate. Clin. Chim. Acta, 33: 293 (1971).

19. Philipps, A. F., Holzman, I. R., Teng, C., and Battaglia, F. C.: Tissue concentrations of free amino acids in term human placentas. Am. J. Obstet. Gynecol. 131(8): 881 (1978).

20. Rice, P. A., Rourke, J. E., and Nesbitt, R. L.: In vitro perfusion studies of the human placenta. IV. Some characteristics of the glucose transport system in the human placenta. Gynecol. Invest., 7: 213 (1976).

21. Salter, W. T., and Robb, P. D.: Ammonia production by sarcoma: The sparing effect of carbohydrate. Am. J. Cancer, 22: 87 (1934).

22. Smith, C. H., Adcock, E. W. III, Teasdale, F., Meschia, G., and Battaglia, F. C.: Placental amino acid uptake: tissue preparation, kinetics, and preincubation effect. Am. J. Physiol., 224: 558 (1973).

23. Stegink, L. D., Pitkin, R. M., Reynolds, W. A., Filer, L. J. Jr., Boaz, D. P., and Brummel, M. C.: Placental transfer of glutamate and its metabolites in the primate. Am. J. Obstet. Gynecol. 122: 70 (1975).

24. Villee, C. A.: The metabolism of human placenta in vitro. J. Biol. Chem., 205: 113 (1953).

25. Weil-Malherbe, H., and Green, R. H.: Ammonia formation in brain. I. Studies on slices and suspensions. Biochem. J., 61: 210 (1955).

26. This research was supported by National Institutes of Health Grants HD 00781 and HD 00429.

27. Requests for reprints should be addressed to: Frederick C. Battaglia, M.D., Container C-218, 4200 East Ninth Avenue, Denver, CO 80262 (USA).

28. Received for publication January 3, 1978.

29. Accepted for publication March 13, 1978. 